\title{
Mimicking the Binding Sites of Wnt Proteins: Rational Design of Wnt/Fzd-Signaling Modulators
}

\author{
Ana I. Fernández-Llamazares ${ }^{1}$, Kevin C. M. Hermans², Peter Timmerman', \\ and W. Matthijs Blankesteijn ${ }^{2}$ \\ ${ }^{1}$ Pepscan Therapeutics, Zuidersluisweg 2, 8243 RC, Lelystad; ${ }^{2}$ Dept. of Pharmacology, University of \\ Maastricht, Universiteitssingel 50, 6229 ER, Maastricht, The Netherlands
}

\section{Introduction}

The interaction between Wnt proteins and Frizzled (Fzd) receptors is a key event that activates all the Wnt signaling pathways [1]. Aberrant regulation of Wnt signaling is linked to a variety of diseases, and compounds that interfere with Wnt/Fzd interactions are potentially useful for their diagnosis and therapeutics.

The aim of the present work was to develop peptides that bind to Fzds, and are able to modulate $\mathrm{Wnt} /$ Fzd-signaling. In order to develop such compounds, we sought to mimic the Fzd-binding sites of Wnt proteins. Although there is limited structural information on the features of Wnt/Fzd interactions, it is likely that all Wnt proteins interact with Fzds through their $\beta 2$ - and $\beta 3$-loop regions [2]. These regions are highly conserved among the different Wnts, and their secondary structure is stabilized by a characteristic pattern of intraloop SS-bonds.

Herein we report the design, synthesis and biological evaluation of a library of peptide mimics for the $\beta 2$ - and $\beta 3$-loop regions of Wnt3a and Wnt5a.

\section{Results and Discussion}

Peptide design: We designed a library of peptides derived from the sequences of Wnt3a and Wnt5a in their their $\beta 2$ - and $\beta 3$-loop regions (Tables 1 and 2). The designed mimics have the same pattern of SS-bonds as the natural Wnt ligands, and are further conformationally constrained via cyclization, for which two additional Cys were introduced at the $\mathrm{N}$ - and $\mathrm{C}$-terminus of the Wntderived sequence and connected via a bivalent CLIPS scaffold (Figure 1). The variables in our library are the length of the peptide sequence, and the position of the two additional Cys. For some of the designed mimics, we also synthesized: i. linear analogs (Cys replaced by $S \mathrm{Me}-\mathrm{Cys}$ ), ii. scrambled analogs, iii. analogs with the $N$ - and $C$-terminal Cys connected via an SS-bond, iv. mutants lacking SS-bonds (Cys replaced by Ala). Data not shown.

Peptide synthesis: We synthesized the peptides by a non-regioselective approach, employing Acmprotection for those Cys to be connected via an SSbond. For every target peptide, we prepared the corresponding linear precursor on the Rink Amide resin by automated solid-phase peptide synthesis, using standard Fmoc/ ${ }^{\mathrm{Bu}}$ chemistry. After cleavage and purification, we cyclized the peptide via Cys-attachment onto the bivalent CLIPS
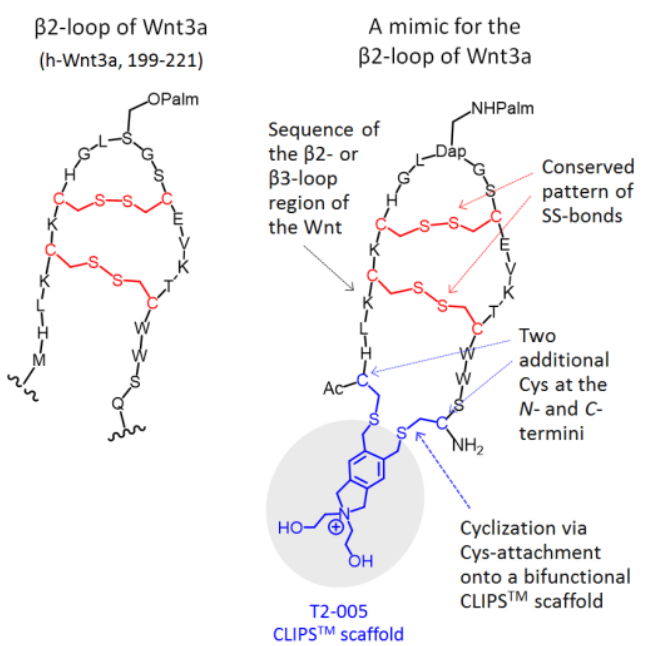

Fig. 1. Schematic representation of a Wntmimicking peptide.

scaffold, and purified the target product. Next, we removed the Acm groups under oxidative conditions $\left(\mathrm{I}_{2}\right)$. In this last synthetic step, all the SS-bonds of the peptide are formed concomitantly, which can afford several SS-bond isomers of unknown SS-bond pattern. For every target peptide, we isolated the SS-bond isomeric products formed, and we tested them separately. 
Table 1. Wnt3a mimics designed, synthesized and tested.

\begin{tabular}{|c|c|}
\hline Peptide & Sequence \\
\hline h-Wnt3a, 199-221 & ${ }^{199} \mathrm{MHLKC}^{\mathrm{B}} \mathrm{KC}^{\mathrm{A}} \mathrm{HGLS}(\mathrm{OPalm}) \mathrm{GSC}^{\mathrm{A}} \mathrm{EVKTC}^{\mathrm{B}}{ }^{\mathrm{B} W S Q^{221}}$ \\
\hline WNT3A-B2-001 & Ac-C ${ }^{\mathrm{T}} \mathrm{HLKCKCHGLDap}(\mathrm{NPalm}) \mathrm{GSCEVKTCWWSC}{ }^{\mathrm{T}}-\mathrm{NH}_{2}$ \\
\hline WNT3A-B2-002 & Ac-C ${ }^{\mathrm{T}}$ LKCKCHGLDap(NPalm)GSCEVKTCWWC ${ }^{\mathrm{T}}-\mathrm{NH}_{2}$ \\
\hline WNT3A-B2-003 & Ac- $\mathrm{C}^{\mathrm{T}}$ KCKCHGLDap(NPalm)GSCEVKTCWC ${ }^{\mathrm{T}}-\mathrm{NH}_{2}$ \\
\hline WNT3A-B2-004 & Ac- $\mathrm{C}^{\mathrm{T}}$ CKCHGLDap(NPalm)GSCEVKTCC ${ }^{\mathrm{T}}-\mathrm{NH}_{2}$ \\
\hline WNT3A-B2-005 & Ac- ${ }^{\mathrm{T}}$ KCHGLDap(NPalm)GSCEVKTC ${ }^{\mathrm{T}}-\mathrm{NH}_{2}$ \\
\hline h-Wnt3a, 312-351 & ${ }^{312} \mathrm{C}^{\mathrm{D}}$ GRGHNARAERRREKC ${ }^{\mathrm{C}} \mathrm{RC}^{\mathrm{B}}{ }^{\mathrm{B}} \mathrm{VHWW} \mathrm{C}^{\mathrm{A}} \mathrm{C}^{\mathrm{A}} \mathrm{YVSC}^{\mathrm{B}} \mathrm{QEC}^{\mathrm{C}} \mathrm{TRVYDVHTC} \mathrm{C}^{\mathrm{D} 351}$ \\
\hline WNT3A-B3-001 & Ac-C ${ }^{\mathrm{T}}$ NARAERRREKCRCVFHWCCYVSCQECTRVYDVHTC ${ }^{\mathrm{T}}-\mathrm{NH}_{2}$ \\
\hline WNT3A-B3-002 & Ac-C ${ }^{\mathrm{T}}$ REKCRCVFHWCCYVSCQECTRVC ${ }^{\mathrm{T}}-\mathrm{NH}_{2}$ \\
\hline WNT3A-B3-003 & Ac-C ${ }^{\mathrm{T}} \mathrm{RCVFHWCCYVSCQEC}{ }^{\mathrm{T}}-\mathrm{NH}_{2}$ \\
\hline WNT3A-B3-004 & Ac-C ${ }^{\mathrm{T}}$ ERREKCRCVFHWCCYVSCQECTRVC ${ }^{\mathrm{T}}-\mathrm{NH}_{2}$ \\
\hline WNT3A-B3-005 & Ac-C ${ }^{\mathrm{T}}$ REKCRCVFHWCCYVSCQECTRC ${ }^{\mathrm{T}}-\mathrm{NH}_{2}$ \\
\hline WNT3A-B3-006 & Ac-C ${ }^{\mathrm{T}}$ REKCRCVFHWCCYVSCQECTC ${ }^{\mathrm{T}}-\mathrm{NH}_{2}$ \\
\hline WNT3A-B3-007 & Ac-C ${ }^{\mathrm{T}}$ EKCRCVFHWCCYVSCQECC ${ }^{\mathrm{T}}-\mathrm{NH}_{2}$ \\
\hline WNT3A-B3-008 & Ac-C ${ }^{\mathbf{T}}$ ARCVFHWCCYVSCQEC ${ }^{\mathrm{T}}-\mathrm{NH}_{2}$ \\
\hline WNT3A-B3-009 & Ac-C ${ }^{\mathrm{T}} \mathrm{RCVFHWCCYVSCQC}{ }^{\mathrm{T}}-\mathrm{NH}_{2}$ \\
\hline WNT3A-B3-010 & Ac- $C^{\mathbf{T}}$ CVFHWCCYVSCC ${ }^{\mathbf{T}}-\mathrm{NH}_{2}$ \\
\hline WNT3A-B3-011 & Ac- $C^{\mathrm{T}}$ VFHWCCYVSC ${ }^{\mathrm{T}}-\mathrm{NH}_{2}$ \\
\hline
\end{tabular}

Nomenclature: Dap $(N P a l m)=N$ - $\beta$-palmitoyl-L-2,3-diaminopropionic acid $; C T=C y$ s connected via the bivalent CLIPS scaffold; $C=$ Cys connected via an SS-bond.

Table 2. Wnt5a mimics designed, synthesized and tested.

\begin{tabular}{|c|c|}
\hline Peptide & Sequence \\
\hline h-Wnt5a, 234-256 & ${ }^{234} \mathrm{ADVAC}^{\mathrm{B}} \mathrm{KC}^{\mathrm{A}} \mathrm{HGVS}(\mathrm{OPalm}) \mathrm{GSC}^{\mathrm{A}}{ }^{\mathrm{S}} \mathrm{KKTC}^{\mathrm{B}} \mathrm{WLQL}^{256}$ \\
\hline WNT5A-B2-001 & Ac-C ${ }^{\mathrm{T}}$ DVACKCHGVDap(NPalm)GSCSLKTCWLQC ${ }^{\mathrm{T}}-\mathrm{NH}_{2}$ \\
\hline WNT5A-B2-002 & Ac-C ${ }^{\mathrm{T}}$ VACKCHGVDap(NPalm)GSCSLKTCWLC ${ }^{\mathrm{T}}-\mathrm{NH}_{2}$ \\
\hline WNT5A-B2-003 & Ac-C $^{\mathbf{T}}$ ACKCHGVDap(NPalm)GSCSLKTCW ${ }^{\mathrm{T}}-\mathrm{NH}_{2}$ \\
\hline WNT5A-B2-004 & Ac-C ${ }^{\mathbf{T}} \mathbf{C K C H G V D a p ( N P a l m ) G S C S L K T C C}{ }^{\mathrm{T}}-\mathrm{NH}_{2}$ \\
\hline WNT5A-B2-005 & Ac- $C^{\mathrm{T}}$ KCHGVDap(NPalm)GSCSLKTC ${ }^{\mathrm{T}}-\mathrm{NH}_{2}$ \\
\hline h-Wnt5a, 340-379 & ${ }^{340} \mathrm{C}^{\mathrm{D}}$ GRGYDQFKTVQTERC ${ }^{\mathrm{C}} \mathrm{HC}^{\mathrm{B}} \mathrm{KFHWC}^{\mathrm{A}} \mathrm{C}^{\mathrm{A}} \mathrm{YVKC}^{\mathrm{B}} \mathrm{KKC}^{\mathrm{C}} \mathrm{TEIVDQFV} \mathrm{C}^{\mathrm{D} 379}$ \\
\hline WNT5A-B3-001 & Ac-C DQFKTVQTERCHCKFHWCCYVKCKKCTEIVDQFVC ${ }^{\mathrm{T}}-\mathrm{NH}_{2}$ \\
\hline WNT5A-B3-002 & Ac-C ${ }^{\mathrm{T}}$ TERCHCKFHWCCYVKCKKCTEIC ${ }^{\mathrm{T}}-\mathrm{NH}_{2}$ \\
\hline WNT5A-B3-003 & Ac- C $^{\mathrm{T}} \mathrm{HCKFHWCCYVKCKKC} \mathrm{C}^{\mathrm{T}}-\mathrm{NH}_{2}$ \\
\hline WNT5A-B3-004 & Ac-C $^{\mathrm{T}}$ VQTERCHCKFHWCCYVKCKKCTEIC ${ }^{\mathrm{T}}-\mathrm{NH}_{2}$ \\
\hline WNT5A-B3-005 & Ac-C ${ }^{\mathrm{T}} \mathrm{QTERCHCKFHWCCYVKCKKCTEC}{ }^{\mathrm{T}}-\mathrm{NH}_{2}$ \\
\hline WNT5A-B3-006 & Ac-C ${ }^{\mathrm{T}}$ TERCHCKFHWCCYVKCKKCTC ${ }^{\mathrm{T}}-\mathrm{NH}_{2}$ \\
\hline WNT5A-B3-007 & Ac-C ${ }^{\mathrm{T}}$ ERCHCKFHWCCYVKCKKCC ${ }^{\mathrm{T}}-\mathrm{NH}_{2}$ \\
\hline WNT5A-B3-008 & 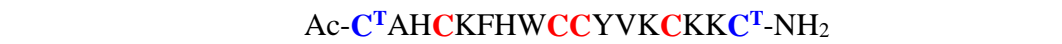 \\
\hline WNT5A-B3-009 & Ac- ${ }^{\mathrm{T}} \mathrm{HCKFHWCCYVKCKC}{ }^{\mathrm{T}}-\mathrm{NH}_{2}$ \\
\hline WNT5A-B3-010 & Ac- $\mathbf{C}^{\mathbf{T}} \mathrm{CKFHWCCYVKCC}^{\mathrm{T}}-\mathrm{NH}_{2}$ \\
\hline WNT5A-B3-011 & Ac-C ${ }^{\mathrm{T}} \mathrm{KFHWCCYVKC}^{\mathrm{T}}-\mathrm{NH}_{2}$ \\
\hline
\end{tabular}

Nomenclature: Dap $($ NPalm $)=N$ - $\beta$-palmitoyl-L-2,3-diaminopropionic acid $; C^{T}=C y s$ connected via the bivalent CLIPS scaffold; $C=$ Cys connected via an SS-bond. 
Biological evaluation: We tested the peptides for their capacity to inhibit Wnt3a-mediated signaling in Fzd1/2-expressing cells. For this purpose, we used a 3T3 cell line that is specially developed for discovering modulators of canonical Wnt signaling, and expresses the TOPFlash reporter. In our assay, we first added Wnt3a-conditioned medium to the cells to induce canonical Wnt signaling, and afterwards we added the peptides at $10-100 \mu \mathrm{M}$.

Several Wnt-mimicking peptides inhibited Wnt3a-mediated signaling at $10 \mu \mathrm{M}$ (i.e. $>50 \%$ inhibitory activity). Based on their activities, we selected 4 peptides as leads for the development of potent Fzd1/2 antagonists (Table 3, Figure 2). For these 4 peptides, regioselective synthesis of the native SS-bond isomers is currently under investigation.

Table 3. Wnt3a and Wnt5a mimics selected as leads.

\begin{tabular}{ccc}
\hline Peptide & Sequence & $\begin{array}{c}\text { SS-isomers } \\
\text { isolated and tested }\end{array}$ \\
\hline WNT3A-B2-003 & Ac-C ${ }^{\mathrm{T}}$ KCKCHGLDap(NPalm)GSCEVKTCWC ${ }^{\mathrm{T}}-\mathrm{NH}_{2}$ & $\mathrm{a}$ \\
WNT3A-B3-009 & Ac-C ${ }^{\mathrm{T}}$ RCVFHWCCYVSCQC ${ }^{\mathrm{T}}-\mathrm{NH}_{2}$ & $\mathrm{a}, \mathrm{b}, \mathrm{c}$ \\
WNT5A-B2-003 & Ac-C ${ }^{\mathrm{T}}$ ACKCHGVDap(NPalm)GSCSLKTCWC ${ }^{\mathrm{T}}-\mathrm{NH}_{2}$ & $\mathrm{a}, \mathrm{b}$ \\
WNT5A-B3-009 & Ac-C & $\mathrm{a}, \mathrm{b}$ \\
\hline
\end{tabular}

Nomenclature: Dap $(N P a l m)=N$ - $\beta$-palmitoyl-L-2,3-diaminopropionic acid; $C^{T}=C y$ s connected via the bivalent CLIPS scaffold; $C=$ Cys connected via an SS-bond.

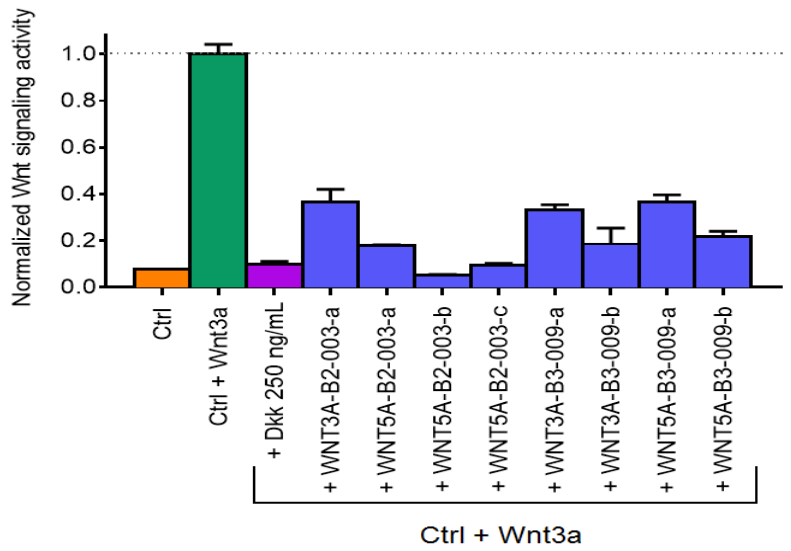

Fig. 2. Inhibitory activities of the Wnt-mimicking peptides selected as leads. when tested at $10 \mathrm{uM}$.
In summary, we have synthesized a library of peptides derived from the sequences of Wnt $3 \mathrm{a} / 5 \mathrm{a}$ in their $\beta 2$ - and $\beta 3$-loop regions. Several of these Wnt mimicking peptides inhibited Wnt3amediated signaling in Fzd1/2expressing cells $(10 \mu \mathrm{M})$, thereby demonstrating that mimicking the Fzdbinding sites of Wnt proteins is a feasible strategy for discovering Wnt/Fzd-signaling modulators.

\section{Acknowledgments}

This work was supported by the Cyttron II research project (grant FES09088), the Dutch Heart Foundation (grant 2010B196), and the EU FP7 Marie Curie ITN program (grant 608180, 'WntsApp').

\section{References}

1. MacDonald, B., Tamai, K., Xei, H. Dev. Cell 17, 9-26 (2009), http://dx.doi.org/10.1016/j.devcel.2009.06.016

2. Janda, C.Y., Waghray, D., Levin, A.M., Thomas, C., Garcia, K.C. Science 337, 59-64 (2012), http://dx.doi.org/10.1126/science.1222879 